\title{
Rursus
}

Ruksus

Poiétique, réception et réécriture des textes antiques

$2 \mid 2007$

Le modèle animal (II)

\section{Conversation et argumentation dans le Banquet de Xénophon}

Résumé de mémoire de linguistique grecque sous la direction de Mlle M.

Biraud (2004-2006)

\section{Pascale Billaud}

\section{(2) OpenEdition}

Journals

Édition électronique

URL : http://journals.openedition.org/rursus/145

DOI : $10.4000 /$ rursus. 145

ISSN : 1951-669X

Éditeur

Université Nice-Sophia Antipolis

Référence électronique

Pascale Billaud, "Conversation et argumentation dans le Banquet de Xénophon », Rursus [En ligne], 2 I 2007, mis en ligne le 04 mai 2007, consulté le 22 septembre 2020. URL : http://

journals.openedition.org/rursus/145; DOI : https://doi.org/10.4000/rursus.145

Ce document a été généré automatiquement le 22 septembre 2020.

Rursus 


\section{Conversation et argumentation dans le Banquet de Xénophon}

Résumé de mémoire de linguistique grecque sous la direction de Mlle M. Biraud (2004-2006)

\section{Pascale Billaud}

1 Nombre de critiques modernes nourrissent une opinion fort négative à l'encontre de Xénophon en qui ils ne voient qu'un piètre imitateur du grand Platon, qu'un disciple à « l'esprit tellement naïf et tellement étroit, ou si philistin, qu'il ne saurait avoir saisit le noyau ou la profondeur de la pensée de Socrate $»^{1}$, son maitre. Ils lui reprochent également son style trop simple, trop naïf, beaucoup moins brillant que celui de Platon. Ces critiques considèrent en effet que le Banquet de Xénophon n'est qu'un piètre assemblage de conversations de salon qui n'entretiennent entre elles aucun lien logique, sérieux.

2 Ce mémoire vise donc à discréditer l'opinion défavorable de ces commentateurs en menant une étude approfondie du Banquet de Xénophon. En réalité cette œuvre « forme vraiment un tout organisé souvent avec une réelle habileté $»^{2}$, elle possède une organisation profonde et sa composition "ne s'en va pas au hasard et comme à la dérive $»^{3}$. Contrairement à ce qu'avance la critique moderne, Xénophon a réussi, dans son Banquet, à rendre avec finesse toutes les nuances et subtilités d'une discussion qui, sous des dehors légers de conversations de salon, traite de manière argumentée de thèmes moraux fort sérieux.

3 Ce mémoire a donc eu pour enjeu d'examiner la nature du rôle qu'occupent respectivement conversation et argumentation dans le Banquet de Xénophon, et de s'interroger sur le lien existant entre ces deux concepts: évoluent-ils de manière indépendante ou ne cessent-ils d'interférer? Autrement dit, il pose la question de savoir si la conversation légère que Xénophon dit nous rapporter ne serait pas soustendue par une immense argumentation implicite visant au profit moral des convives...

4 Notre étude suivra quatre étapes. Nous commencerons par présenter l'ensemble des informations nécessaires (contexte et différents domaines conceptuels) au développement d'une analyse approfondie du Banquet. Puis nous nous livrerons 
successivement à trois études suivies portant chacune sur un chapitre de l'œuvre : ces derniers seront choisis de manière à présenter à chaque fois une facette argumentative ou conversationnelle différente afin que l'ensemble reflète l'essentiel des caractéristiques argumentatives et conversationnelles de l'œuvre. Nous étudierons donc tout d'abord le troisième chapitre où l'aspect conversationnel parait dominer : chacun des convives doit exposer l'objet dont il se sent le plus fier, ce qui entraîne à chaque fois plusieurs commentaires... Nous examinerons ensuite le cinquième chapitre qui, inversement, paraît essentiellement privilégier la dimension argumentative : il s'agit d'une joute argumentative entre Socrate et Critobule au sujet de la beauté. Enfin, nous nous tournerons vers un passage du huitième chapitre: Socrate y souhaite démontrer la supériorité de l'amour de l'âme sur celui du corps. Contrairement au chapitre précédemment analysé, celui-ci se présente d'emblée comme une argumentation sérieuse, reposant sur une structure solide et non sophistique.

Nous en arriverons aux conclusions suivantes. Bien qu'ils soient d'aspects fort distincts, ces trois passages nous permettent de constater que, loin d'être « une composition très décousue, surchargée de hors d'œuvres $»^{4}$, l'œuvre possède une réelle unité d'ensemble. Ces trois chapitres s'insèrent en effet dans une logique thématique d'ensemble, assurant la cohérence globale de l'œuvre, mais il convient de remarquer également que du point de vue interne, leur architecture est également fort travaillée, et non laissée au hasard... Xénophon est donc capable de présenter des discussions qui, sous des dehors parfois légers et désordonnés, possèdent une cohérence et une structure interne d'une grande précision. Mais c'est également dans l'observation des relations interpersonnelles que l'écriture de Xénophon s'est révélée d'une sensibilité et d'une richesse remarquables : à l'image d'un Thucydide ou, à une époque postérieure, d'un Théophraste, qui tous deux s'appliquaient à rendre avec finesse les méandres de la conscience humaine, l'auteur du Banquet a su se faire l'observateur des comportements humains dans toute leur complexité... Ainsi, respect ou manquement aux règles de politesse, profils conversationnels et surtout importance du jeu sur l'implicite témoignent de la virtuosité et de la finesse de Xénophon. Ajoutons que ce concept d'implicite est au centre de l'œuvre puisqu'il joue un rôle non négligeable dans les divers systèmes argumentatifs. Xénophon nous donne également à voir la virtuosité oratoire dont Socrate était capable dans ses divers discours. L'auteur réalise donc une brillante démonstration de son habileté à mettre en scène des discours argumentatifs s'appuyant sur la rhétorique. Ce qui nous amène une fois de plus à remettre en cause l'opinion des critiques avançant que Xénophon était incapable de témoigner dans ses écrits des méandres et de la complexité de la pensée de son maître... Devant une telle variété d'utilisation de procédés stylistiques, nous avons peine à croire que l'écriture de Xénophon soit trop simple, sans profondeur et peu ravissante. Mais surtout en nous présentant des argumentations ayant toutes leur efficacité, bien que certaines jouent en apparence sur la provocation, le manque de sérieux et la mauvaise foi, alors que d'autres sont au contraire caractérisées par la rigueur des raisonnements, Xénophon nous prouve qu'il n'est nul besoin d'austérité ou de pédantisme pour accéder à la connaissance et à la sagesse : celle-ci peut être dévoilée à travers des actions plaisantes et des conversations d'apparence légère mêlant la gaîté, le jeu et la bonne humeur... Ce dernier point nous aura permis de constater que dans le Banquet, conversation et argumentation étaient intimement liées : la conversation à laquelle Socrate impose plus ou moins discrètement ses orientations, contient en filigrane une immense argumentation en faveur de la kalokagathia et des moyens d'y accéder. 
6 Enfin, si Xénophon ne nous présente pas un Socrate en tous points identique à celui de Platon, ce n'est pas parce qu'il n'a rien compris à la pensée de son maître, mais parce qu'il souhaite décrire ce qui, selon lui, caractérisait le mieux le caractère et les manières de procéder du philosophe.

\section{NOTES}

1. L. Strauss, 1992, Introduction au Discours Socratique de Xénophon, Combas, éd. de L'Eclat.

2. F. Ollier, 2002 [1961], Introduction, Xénophon : Banquet, Paris, CUF, p.10.

3. F. Ollier, 2002 [1961], p.10.

4. L. Robin, 1970, Platon : Banquet, Notice, Paris, CUF, p. CXI. 